\title{
Stemi in women
}

\author{
Lekha Pathak, Salil Shirodkar, Jaideep Rajebahadur, Sachinkumar Patil and Ankur Jhavar \\ Nanavati Superspeciality Hospital, Mumbai, India
}

\begin{abstract}
Background: Cardiovascular disease (CVD) is the leading cause of mortality in Indian women. Acute coronary syndromes (ACS), including unstable angina (UA), non-ST-segment elevation myocardial infarction (NSTEMI), and ST-segment elevation myocardial infarction (STEMI), represent a large portion of the clinical presentation of coronary artery disease (CAD) in Indians. Despite the importance of CAD for women, there is persistent perception that CAD is a man's disease. We have been interested in coronary artery disease in women and thereafter we observed interesting data about STEMI in women, particularly highlighting the incidence, risk factors, interventional procedure with immediate and long-term outcome.

Methods: We studied clinical and angiographic profiles of 100 women admitted with STEMI who underwent coronary angiography and angioplasty over a period of 5 years at Nanavati Hospital, Mumbai, India. The objectives were to examine the distribution of risk factor and coronary angiographic patterns of CAD in women presenting with STEMI.

Results: It was observed that myocardial infarction is most commonly involving women between the age 50 to 80 years. Diabetes mellitus and Hypertension were found to be most common risk factors involved in development of myocardial infarction in obese females. Most common presentation of STEMI was inferior wall MI. Most common coronary angiography finding was multi vessel disease.

Conclusion: The clinical presentation of myocardial infarction in women varies from mild chest discomfort to severe unstable angina. Primary angioplasty is the gold standard treatment for such patients. Data about gender as an independent prognostic factor for death is conflicting. Women usually present at a later age with atypical symptoms and have higher rates of comorbidity, which translates to a worse prognosis post-ACS than men. As this study is based on experience at single centre, various biases may be possible.
\end{abstract}

\section{Introduction}

Coronary artery disease has traditionally been considered a disease of men, but what has been the odyssey for women in the century since its initial description by Herrick in 1912? Despite stunning improvements in cardiovascular mortality for women in the past 2 decades, CAD remains understudied, underdiagnosed, and undertreated in women. The worldwide INTERHEART Study, a large cohort study of more than 52000 individuals with myocardial infarction, have revealed that women have their first presentation of coronary heart disease approximately 10 years later than men, most commonly after menopause [1]. Despite this delay in onset, mortality is increasing more rapidly amongst women than men. Cardiovascular disease (CVD) is the leading cause of mortality for women in the India and globally [2]. It accounts for 1 out of 3 women death regardless of the race or ethnicity [3]. From 1960 to 1995, the prevalence of CAD in adults increased from $3 \%$ to $10 \%$ in urban Indians and from $2 \%$ to $4 \%$ in rural Indians with women having rates similar to men [4]. Women's health involves 2 aspects: sex differences resulting from biological factors and gender differences affected by broader social, environmental, and community factors [5]. Although the emphasis on sex-specific CVD research for the past 2 decades has led to an improved understanding of sex-specific pathophysiology for CHD in women and important insights into an expanded spectrum of coronary atherosclerosis, female research subjects are underrepresented when studies are designed, conducted, and analysed [6]. Multiple studies have shown that women with acute coronary syndromes (ACS) are less likely to be treated with guideline-directed medical therapies, less likely to undergo cardiac catheterization, and less likely to receive timely reperfusion $[7,8]$.
Our study is a single center study based on all women presenting with STEMI. We studied clinical and angiographic profile of women with STEMI undergoing angiography at our center in 5 years. This study provides in depth understanding about distribution of CAD amongst various age group of women and its possible cause. It also compares various risk factors, clinical presentation and angiographic picture of women undergoing coronary angiography and angioplasty for STEMI and their 6 monthly follow-up.

\section{Cad Mortality in Women}

In Framingham heart study, the one-year mortality following an MI was $44 \%$ in women vs. $27 \%$ in men. The excess in hospital CAD mortality in women compared to men almost balances their lower prehospital mortality [9]. Even among women who undergo PCI, they experience a higher rate of complications and mortality than men [10]. Women are more likely than men to die after a first MI, and for survivors, there is higher risk of recurrent MI, heart failure or death. Vaccarino et al. found that mortality from MI in women $<50$ years of age was double that of men and excess mortality in women is limited to $<60$ years of age [11].

${ }^{\star}$ Correspondence to: Pathak L Nanavati Superspeciality Hospital, Mumbai, India, Email: drlekha_p@yahoo.co.in

Key words: Cardiovascular disease, mortality, Acute coronary syndromes

Received: May 04, 2018; Accepted: June 27, 2018; Published: June 30, 2018 


\section{Risk Factors in Women}

Women, in comparison with men, tend to have a better risk factor profile at younger ages, whereas the opposite is true at older ages. Women are about 10 years older than men at first manifestation of $\mathrm{CAD}$, although they have similar plaque burden [12]. Average age of first myocardial infarction has decreased by 20 years in India. STEMI among Indians is at least double that of Americans and several folds higher than other Asians [13]. Among women, a history of an MI or sudden death before the age of 55 in a sister is more strongly associated with risk of MI than that in a brother or parent. Among Indian women, the presence of hypertension, diabetes, low levels of high density lipoprotein and high levels of total cholesterol, triglycerides, low-density lipoprotein and Lp (a) are correlated with CAD and may lead to myocardial infarction [14]. Diabetes is a stronger risk factor for $\mathrm{CAD}$ in women than in men, with a 3 - to 7 - fold higher CAD incidence and mortality compared to a 2- to 3 - fold higher risk in men $[15,16]$. Obesity is a major risk factor for AMI in women and increases their risk almost 3-fold [17]. Prevalence of most risk factors is lower in rural than in urban India with exception of smoking / tobacco use (Tobacco Paradox) [18]. In the INTERHEART study, an aggregate exposure to psychosocial risk factors, including depression, perceived home, work stress, low locus of control, and major life events, were significantly associated with AMI in women, with an adjusted odds ratio of 3.5 [19].

\section{Patterns of Presentation}

Although most patients with AMI present with typical chest pain or chest discomfort, women often present with atypical chest pain and angina equivalent symptoms such as dyspnea, weakness, fatigue, and indigestion [20]. Compared with men, women are more likely to have high risk presentations and less likely to manifest central chest pain $[21,22]$. Pain in the upper back, arm, neck, and jaw, as well as unusual fatigue, dyspnea, indigestion, nausea/vomiting, palpitations, weakness, and a sense of dread, occur more frequently in women compared with men [23].

\section{Aims and Objectives}

Our study aimed at studying clinical profile of women presenting with STEMI at our center. We studied various risk factors and their association with occurrence of myocardial infarction in 100 Women admitted with STEMI over a period of 5 years. Our study tried to determine age distribution and possible reason for the same. We also compared various angiographic patterns amongst women undergoing coronary angiography for myocardial infarction. Inspect your feet twice daily. Look all over the feet for cracks, blisters, reddened spots, cuts, and ulcers or for excessively moist skin between the toes.

\section{Study Profile}

In this retrospective analysis of 100 women, who underwent coronary angiography and angioplasty for STEMI during past 5 years at our centre. We studied various clinical presentations and the risk factors along with the angiographic profile. The decision for PCI was made by the operator. The risk factor profile was studied in terms of presence of hypertension, diabetes mellitus, dyslipidemia, smoking, metabolic syndrome, physical inactivity and family history.

In our analysis we found the STEMI was most prevalent between the age group 50-80 years (Figure 1). Out of 100 females, 52\% were post-menopausal, $30 \%$ were pre-menopausal and $18 \%$ were post hysterectomy. Total $42 \%$ females were vegetarian in our study population, whereas $58 \%$ was having mixed diet. Out of females

\section{Age Distribution}

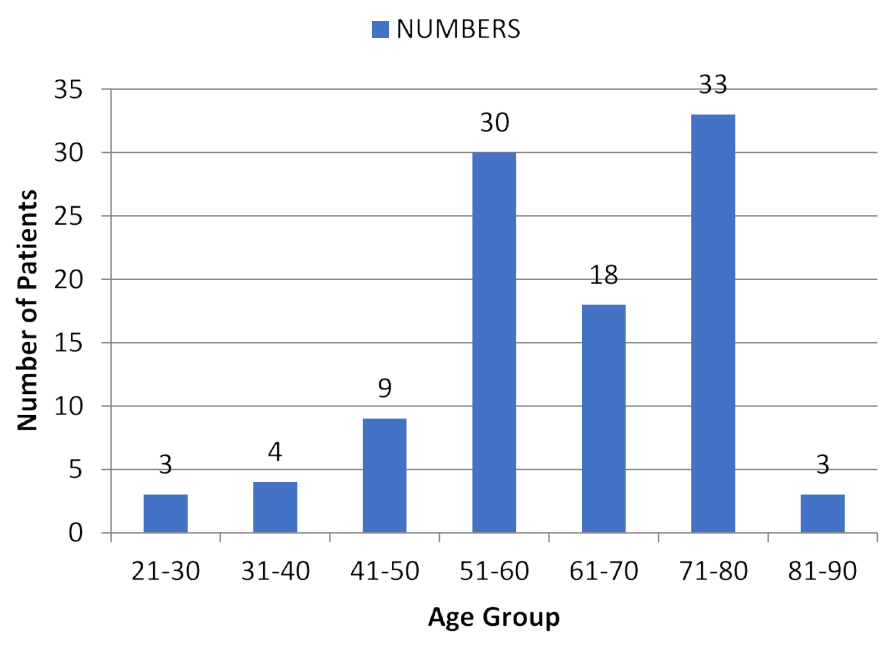

Figure 1. STEMI prevalance between the age group 50-80 years.

consuming mixed diet, $36 \%$ eats red meat and rest $64 \%$ did not. The risk factor profile revealed association of Diabetes mellitus (70\%), Hypertension (65\%) and obesity (57\%), followed in turn by family history (54\%) and dyslipidemia (48\%) (Figure 2). Active smoking was present only in $4 \%$ patients. The family history of Coronary artery disease (in 1st degree relatives) was found to be present in $44 \%$ of women. ECHO was suggestive mainly of poor LV function (86 patients) with regional wall motion abnormalities in almost all of them. Most common presentation of STEMI was inferior wall myocardial infarction (IWMI) (42\%) followed by anterior wall myocardial infarction (AWMI) (30\%) (Figure 3). Other rare presentation was of anterolateral myocardial infarction (19\%) and posterior wall myocardial infarction (PWMI) (9\%) (Figure 3). Analysis of coronary angiographic findings showed that women most commonly suffered from multi vessel disease (75\%) followed by single vessel disease (25\%) (Figure 4). Single vessel disease is rare in women as compared to men. Women usually have associated disease of the other arteries. This set of patients carried a bad prognosis during or after intervention in our group. Out of total 156 lesions, $61 \%$ were Type A, 27\% were Type B and $12 \%$ were Type C. Calcification was found to be associated with 28 lesions. Left main disease was found in 6 females whereas ostial lesion in 34 and bifurcation disease was in 22 women. 56 had diffuse lesion involving either one or more coronary arteries.

\section{Conclusion}

Acute myocardial infarction in women continues to be a major public health problem that represents a leading cause of death and disability. They can have a varied presentation ranging from typical chest pain to atypical chest discomfort and angina equivalent symptoms such as dyspnea, weakness, unusual fatigue etc. Early diagnosis is essential and prompt management is effective at reducing mortality and improving outcomes post ACS. Women respond just as well as men to acute in-hospital treatments and post hospital secondary prevention. Age distribution of STEMI amongst women showed that elderly women are more affected, this can be secondary to post menopausal status. To prove estrogen as a protective factor for coronary artery disease, wide spread study with multiple parameters is recommended. Various risk factors have been studied in our study, DM, HTN and obesity being the commonest. Women have varied 


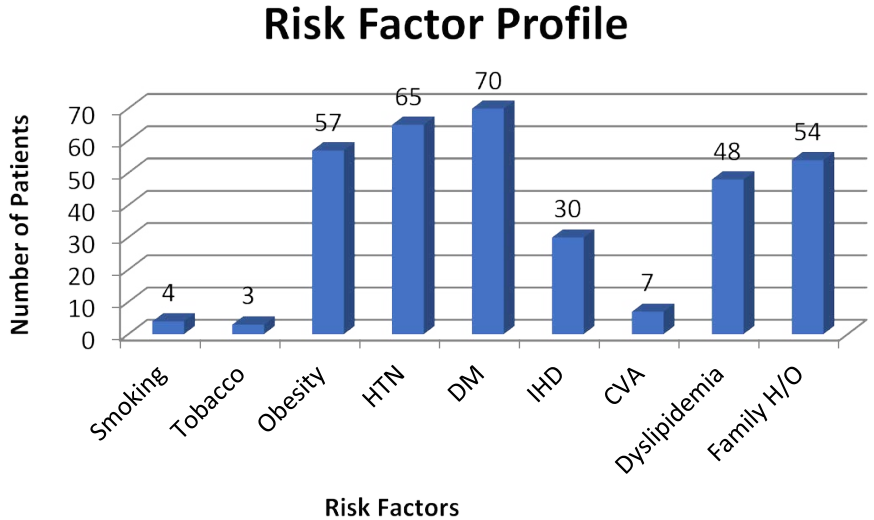

Figure 2. The risk factor profile revealed association of Diabetes mellitus (70\%), Hypertension $(65 \%)$ and obesity $(57 \%)$, followed in turn by family history $(54 \%)$ and dyslipidemia $(48 \%)$

\section{Clinical Presentation}

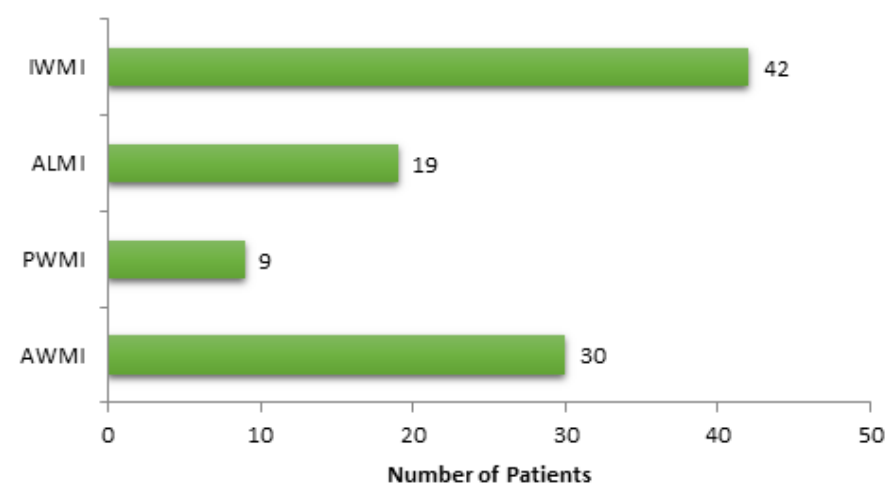

Figure 3. Most common presentation of STEMI was inferior wall myocardial infarction (IWMI) (42\%) followed by anterior wall myocardial infarction

\section{Angiographic Profile}

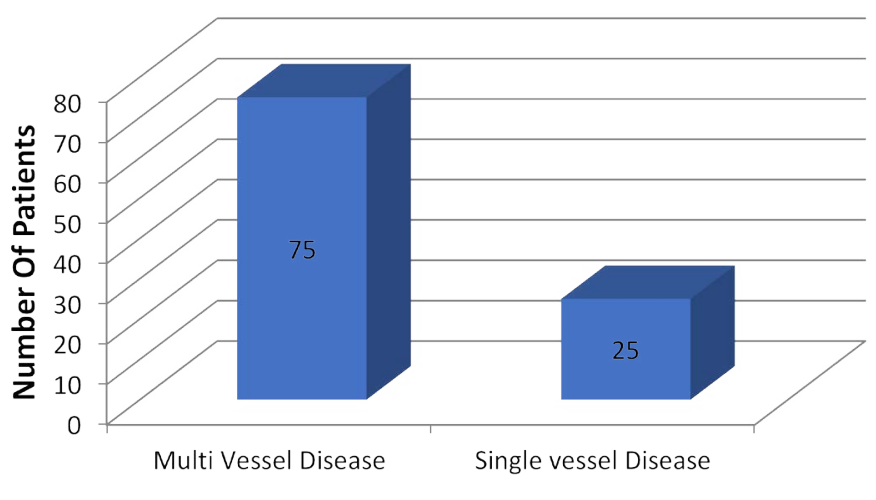

Figure 4. Analysis of coronary angiographic findings

presentation angiographically from single vessel disease to multi vessel disease in which characteristics of coronary lesion also vary. Women are undertreated with guideline-based recommendations, leading to worse outcomes and increased rate of readmission, reinfarction, and deaths in the first year after MI. To further compound undertreatment, women's adherence to these evidence-based recommendations is suboptimal. There is a need for continued public health messages and interventions to target racial and ethnic minority women, given the burden of risk factors and continued disparity in outcomes. There should be increased awareness of women, healthcare providers, the public, and policymakers of MI risk and sex-specific symptoms and clinical presentation. Evaluate the mechanisms by which psychosocial risk factors (e.g. depression, perceived stress, marital conflict, anxiety, poor social support) influence CAD development and progression. Offer sexual counselling to all women and their partners before hospital discharge after ACS. Implement effective psychological treatment to reduce barriers and to improve adherence to guidelinebased recommendations and to improve quality of life. More and more females are presenting with AMI at younger age, partly due to life style disease as well as strong genetic factors. Hypertension and Diabetes are equally contributing for the same. Hence, we need to develop strategies to increase the inclusion of women of all ages in cardiovascular clinical research.

\section{Referrences}

1. Wenger NK (2012) Women and coronary heart disease: a century after Herrick: understudied, underdiagnosed, and undertreated. Circulation 126: 604-611. [Crossref]

2. Gholizadeh L, Davidson P (2008) More similarities than differences: an international comparison of CVD mortality and risk factors in women. Health Care Women Int 29 3-22. [Crossref]

3. Thom T, Hasse N, Rosamond W (2006) Heart disease and stroke statistics- 2006 update: A report from the American Heart Association Statistics Committee and Stroke Statistics Subcommittee. Circulation 113: e85. [Crossref]

4. Gupta R (2016) Coronary heart disease epidemiology in India: the past, present and future. Ann Glob Health 82: 307-315. [Crossref]

5. Wizemann TM, Pardue M (2001) Understanding the Biology of Sex and Gender Differences. Exploring the Biological Contributions to Human Health: Does Sex Matter? The National Academies Press, Washington, USA.

6. Institute of Medicine. Women's Health Research: Progress, Pitfalls, and Promise. Washington, DC: National Academies Press; 2010.

7. Merz CN (2011) The Yentl syndrome is alive and well. Eur Heart J 32: 1313-1315. [Crossref]

8. Blomkalns AL, Chen AY, Hochman JS, Peterson ED, Trynosky K, et al. (2004) National Quality Improvement Initiative. J Am Coll Cardiol. 45: 832-837. [Crossref]

9. Mosca L, Manson J (1997) cardiovascular disease in women. Circulation 96: 2468 2482.

10. Fang J, Alderman MH (2006) Gender differences of revascularization in patients with acute myocardial infarction. Am J Cardiol 97: 1722-1726. [Crossref]

11. Vaccarino V, Harlan (1995) sex differences in mortality after myocardial infarction: is there evidence for an increased risk for women? Circulation 91: 1861-1871. [Crossref]

12. Kornowski R, Lansky AJ, Mintz GS, Kent KM, Pichard AD, et al. (1997) Atherosclerotic Plaque Burden and CK-MB Enzyme Elevation After Coronary Interventions. Am J Cardiol 79: 1601-1605. [Crossref]

13. Forouhi N, McKeigue P (1997) How far can risk factors account for excess coronary mortality in South Asians? Can J Cardiol 13:47.

14. Gupta R, Puri V, Narain V (1999) Cardiovascular risk profile in Indian women. Indian Heart J 51: 679 .

15. Barrett-Connor EL, Cohn BA, Wingard DL, Edelstein SL (1991) The Rancho Bernardo Study. JAMA 265: 627-631

16. Sowers JR (1997) Diabetes mellitus and cardiovascular disease in women. Arch Intern Med 1998; 158: 617-621. [Crossref]

17. Kanaya AM, Grady D, Barrett-Connor E (2002) Explaining the sex difference in coronary heart disease mortality among patients with type 2 diabetes mellitus: a metaanalysis. Arch Intern Med 162: 1737-1745. [Crossref]

18. Reddy KS, Shah P, Prabhakaran P, Shah B, Prabhakar A, et al. (1997) Coronary heart disease risk factors in an industrial population in North India. Indian Heart J 49: 659. 
19. Yusuf S, Hawken S, Ounpuu S, Dans T, Avezum A, et al. (2004) Interheart Study Investigators. Lancet 364: 937-952.

20. Wenger NK (2010) Angina in women. Curr Cardiol Rep. 12: 307-314.

21. Khan NA, Daskalopoulou SS, Karp I, Eisenberg MJ, Pelletier R, et al. (2013) Sex differences in acute coronary syndrome symptom presentation in young patients. JAMA Intern Med. 173: 1863-1871. [Crossref]
22. McSweeney JC, Cleves MA, Zhao W, Lefler LL, Yang S (2010) Cluster analysis of women's prodromal and acute myocardial infarction symptoms by race and other characteristics. J Cardiovasc Nurs 25: 311-322. [Crossref]

23. DeVon HA, Ryan CJ, Ochs AL, Shapiro M (2008) Symptoms across the continuum of acute coronary syndromes: differences between women and men. Am J Crit Care 17: 14-24. [Crossref]

Copyright: $\odot 2018$ Pathak L. This is an open-access article distributed under the terms of the Creative Commons Attribution License, which permits unrestricted use, distribution, and reproduction in any medium, provided the original author and source are credited. 